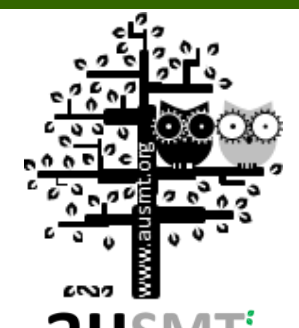

\title{
Automatic Inspection and Processing Based on Vision Stitching and Spectral Illumination
}

\section{Wen-Yang Chang* and Chih-Ping Tsai}

Department of Mechanical and Computer-Aided Engineering, National Formosa University, Taiwan (Received 13 January 2014; Accepted 8 April 2014; Published 1 September 2014)

*Email: wenyang@nfu.edu.tw DOI: 10.5875 /ausmt.v4i3.496

\begin{abstract}
The study investigates the automatic inspection and processing of bicycle components based on vision stitching and spectral illumination. Vision stitching mainly involves the use of algorithms of white balance, scale-invariant feature transform (SIFT) and roundness for automatic accessory inspection of the whole component image. The illumination intensities, angles, and spectral characteristics of light sources are analyzed using a spectrometer. Unrealistic color casts of feature inspection for global automatic adjustment are removed using a white balance algorithm. For the stitching of large images, SIFT is used to extract and detect the image features. The Hough transform is used to detect the roundness of the bicycle component. The inspected features include geometry size, roundness, and image stitching. Results showed maximum errors of $0^{\circ}, 10^{\circ}, 30^{\circ}$, and $50^{\circ}$ for the spectral illumination of white light LED arrays with respective differential shift displacements of 4.4, 4.2, 6.8, and 3.5\%. The deviation errors of image stitching for the stem accessory in $x$ and $y$ coordinates are 2 pixels. SIFT and random sample consensus (RANSAC) enable the transformation of the stem image into local feature coordinates.
\end{abstract}

Keywords: Illumination, automatic inspection, light source, vision stitching

\section{Introduction}

Modern bicycles frequently include as many as 2,000 individual components which must be manufactured with a high degree of precision, thus raising the importance of quality inspections based on non-contact detection and automated manufacturing based on vision stitching.

Most bicycle manufacturers still use contact inspections to detect component defects, and there is an urgent need to develop effective approaches to automate inspection processes. It is difficult for automatic inspections based on image vision to capture a whole component during manufacturing. Vision image stitching based on structures shared between digital images is an important technique for the recognition of large objects. However, image stitching incurs large computational costs and can fail if shared features cannot be identified [1-3]. Most conventional approaches to image stitching are based on scale-invariant feature transforms (SIFT) [4-6] and feature matching [7, 8].

In SIFT image stitching, variations between image features arise not only from changes in the focal viewpoint but also due to spectral illumination [9]. Many theoretical methods based on spectral illumination have been proposed to address this issue, including Bayesian estimation [10], genetic algorithms [12], and feature 
descriptors [13]. One image registration approach based on robust M-estimators [11] compensates for arbitrarily shaped illumination variations.

This study investigates the automatic inspection and processing of bicycle handlebar stems (i.e., the component which holds the handlebars) based on vision stitching and spectral illumination. Images for object recognition are analyzed based on different degrees of spectral illumination and geometric features. Therefore, the angles, intensities, and spectral illumination of light sources are analyzed using a spectrometer. The unrealistic color casts are removed using white balance. The image stitching and the roundness of the stem are automatically assessed using the SIFT and Hough transform, respectively.

\section{Experiment}

The vision images are acquired using a gigabit ethernet (GigE) camera (SLW-DC-GC13M30 FRAMOS, Germany). The light source is 4 frame LEDs placed directly before the object. Prior to the experiment, the spectral illumination of the light source was analyzed in a darkroom and the camera was calibrated using a standard chessboard pattern with a resolution of $10 \mu \mathrm{m}$. Non-contact automatic inspection of the stem accessory used of the white balance, SIFT, and roundness algorithms as follows:

\section{Automatic white balance algorithm}

Wen-Yang Chang is an associate professor in the Department of Mechanical and Computer-Aided Engineering at National Formosa University. He received his Ph.D. from the Department of Engineering Science at National Cheng Kung University in 2008. His main research interests include vision image processing, automatic control systems, digital logical controls, and flexible electronics.

Chih-Ping Tsai received his M.S. from the Department of Mechanical and Computer-Aided Engineering at National Formosa University in 2013. His main research interests include vision digital image processing, automatic control systems, and robot controls.

White balance is a global adjustment to remove unrealistic color casts due to color temperature of a light source. Adjustment of the white balance renders specific colors (particularly neutral colors) and color correction. The gain factors of the gray world assumption algorithm (GWA) can be obtained by simply dividing the gray value by the appropriate average of each color component. The color light can reacquire the true color of the original by re-running the GWA on the image. For this study, automatic GWA for the adjusted colors can expressed as follows:

$$
\left\{\begin{array}{l}
\bar{B}=\frac{G_{a v y} \times I_{B}}{B_{a v g}}=\left(\frac{R_{a v g}+G_{a v g}+B_{a v g}}{3} \times I_{B}\right) /\left(\frac{\sum_{i=0}^{m} \sum_{j=0}^{n} I_{B}}{m \times n}\right) \\
\bar{G}=\frac{G_{a v y_{a v g}} \times I_{G}}{G_{a v g}}=\left(\frac{R_{a v g}+G_{a v g}+B_{a v g}}{3} \times I_{G}\right) /\left(\frac{\sum_{i=0}^{m} \sum_{j=0}^{n} I_{G}}{m \times n}\right) . \\
\bar{R}=\frac{G_{\text {rav }} \times I_{R}}{R_{a v g}}=\left(\frac{R_{a v g}+G_{a v g}+B_{a v g}}{3} \times I_{R}\right) /\left(\frac{\sum_{i=0}^{m} \sum_{j=0}^{n} I_{R}}{m \times n}\right)
\end{array}\right.
$$

where Grayavg represents the gray value and is calculated from the average of the $R, G$, and $B$ color components. $B_{\text {avg, }} G_{\text {avg, }}$ and $R_{\text {avg }}$ are the mean values of $B, G$, and $R$ components, respectively. $I_{R}, I_{G}$, and $I_{B}$ are the original intensities of the $R, G$ and $B$ components, respectively.

\section{SIFT algorithm}

The SIFT algorithm, which mainly consists of descriptors and detectors, is used to conduct object recognition, video tracking, and image stitching [14] by extracting and detecting the image edge features. The descriptors identify the orientation assignments and create the descriptors using histogram orientations. The detectors find the scale-space extrema, keypoint localization, and filter noise. The scale-space extrema, $L(x, y, \sigma)$, is based on Gausscian blur, $G(x, y, \sigma)$, and the difference of Gausscian (DoG), $D(x, y, \sigma)$. The scale-space extrema of an image is defined by Equation (2) [14].

$$
L(x, y, \sigma)=G(x, y, \sigma) * I(x, y)=\frac{1}{2 \pi \sigma^{2}} e^{-\frac{x^{2}+y^{2}}{2 \sigma^{2}}} * I(x, y),
$$

where $\sigma$ signifies the scale factor which determines the width of the kernel and $*$ is a convolution operator. $I(x, y)$ is the intensity level of an input image at the pixel location determined by the $\mathrm{x}$ and $\mathrm{y}$ coordinates.

Scale-space extrema detection produces many keypoint candidates, but some of methods are not good enough for keypoint localization. To determine the keypoint localizations of the scale-space extrema, the $D(X)$ then takes the derivative with respect to $X$ and sets it to 0 after differentiation. Derivatives are approximated by finite differences and, if the contrast value of the second-order Taylor expansion $D(X)$ is below the threshold, the keypoint candidates are discarded. The new keypoint localization of $(\hat{x}, \hat{y}, \hat{\sigma})$ can be written as a matrix as in Equation (3). The $\mathrm{D}(\mathrm{X})$ range is within $[0,1]$ and the keypoint localization is rejected when the low contrast of $D(X)$ is below $0.03[6]$. 


$$
\left[\begin{array}{l}
\hat{x} \\
\hat{y} \\
\hat{\sigma}
\end{array}\right]=-\left[\begin{array}{lll}
\frac{\partial^{2} D}{\partial x^{2}} & \frac{\partial^{2} D}{\partial x \partial y} & \frac{\partial^{2} D}{\partial x \partial \sigma} \\
\frac{\partial^{2} D}{\partial y \partial x} & \frac{\partial^{2} D}{\partial y^{2}} & \frac{\partial^{2} D}{\partial y \partial \sigma} \\
\frac{\partial^{2} D}{\partial \sigma \partial x} & \frac{\partial^{2} D}{\partial \sigma \partial y} & \frac{\partial^{2} D}{\partial \sigma^{2}}
\end{array}\right]^{-1}\left[\begin{array}{l}
\frac{\partial D}{\partial x} \\
\frac{\partial D}{\partial y} \\
\frac{\partial D}{\partial \sigma}
\end{array}\right] .
$$

where

$$
\begin{aligned}
\frac{\partial D}{\partial x}= & \frac{1}{2}[D(x+1, y, \sigma)-D(x-1, y, \sigma)] \\
\frac{\partial^{2} D}{\partial x^{2}}= & D(x+1, y, \sigma)-2 D(x, y, \sigma)+D(x-1, y, \sigma) \\
\frac{\partial^{2} D}{\partial x \partial y}= & \frac{1}{4}[(D(x+1, y+1, \sigma)-D+(x-1, y+1, \sigma) \\
& -D(x+1, y-1, \sigma)+D(x-1, y-1, \sigma)]
\end{aligned}
$$

The Hessian matrix [15] is used to eliminate unstable edge responses and poorly defined peaks according the ratio of principal curvatures. The Hessian matrix, $H$, is based on second-order partial derivatives of a function equation. The trace, $\operatorname{Tr}(\mathrm{H})$, and determinant, $\operatorname{Det}(\mathrm{H})$, of Hessian eigenvalues are proportional to the principal curvatures as in Equation (4). Let $\alpha$ be the eigenvalue with a larger magnitude and $\beta$ is the smaller. In general, we are more concerned with the ratio of $\operatorname{Tr}(\mathrm{H})$ and $\operatorname{Det}(\mathrm{H})$ rather than the actual values of Hessian eigenvalues. We assume $r=\alpha / \beta$ and use $r=10$. Then, Equation (5) is used to determine if the ratio of principal curvatures, $R_{h}$, is below a certain threshold.

$$
\begin{gathered}
\left\{\begin{array}{l}
\operatorname{Tr}(H)=\frac{\partial^{2} D}{\partial x^{2}}+\frac{\partial^{2} D}{\partial y^{2}}=\alpha+\beta \\
\operatorname{Det}(H)=\frac{\partial^{2} D}{\partial x^{2}} \frac{\partial^{2} D}{\partial y^{2}}-\frac{\partial^{2} D}{\partial x \partial y} \frac{\partial^{2} D}{\partial y \partial x}=\alpha \beta
\end{array}\right. \\
R_{H}=\frac{\operatorname{Tr}(H)^{2}}{\operatorname{Det}(H)}=\frac{(\alpha+\beta)^{2}}{\alpha \beta}=\frac{(r \beta+\beta)^{2}}{r \beta^{2}}=\frac{(r+1)^{2}}{r} .
\end{gathered}
$$

The keypoint descriptor can be represented by the descriptor distance orientation and therefore achieve invariance to image rotation when an orientation is assigned to each keypoint. The gradient magnitude, $m(x, y)$, and orientation, $\theta(x, y)$, of an image $L(x, y)$ at scale are computed using pixel differences as in Equation (6). The orientation histogram is formed with 36 bins, with each bin covering 10 degrees.

$$
\left\{\begin{array}{l}
m(x, y)=\sqrt{\left(\frac{\partial L}{\partial x}\right)^{2}+\left(\frac{\partial L}{\partial y}\right)^{2}}=\sqrt{[L(x+1, y)-L(x-1, y)]^{2}+[L(x, y+1)-L(x, y-1)]^{2}} \\
\theta(x, y)=\tan ^{-1}\left(\frac{\partial L}{\partial y} / \frac{\partial L}{\partial x}\right)=\tan ^{-1}\left(\frac{L(x, y+1)-L(x, y-1)}{L(x+1, y)-L(x-1, y)}\right)
\end{array}\right.
$$

The SIFT descriptor is then built for 3-D spatial histogram of the image gradients over small local areas. The histogram gradients at each pixel are regarded as a sample of a 3-D elementary feature vector and provide some measurement of invariance to small changes in keypoint localization. The global normalization of the descriptor distance between $a$ and $b, D(a, b)$, is shown in Equation (7). Let $D_{c}$ and $D_{s}$ respectively be the closest neighbor and second-closest neighbor of the Euclidean distance. For recognition accuracy, a ratio of $D_{c} / D_{s}$ is defined as a threshold $D_{T}$. In general, the recognition accuracy rapidly increases when the threshold is below $0.8[14]$

$$
\left\{\begin{array}{l}
D(a, b)=\sum_{m=1}^{M} \sum_{i=1}^{N}\left|a_{m}[i]-b_{m}[i]\right| \\
\frac{D_{c}}{D_{s}}<D_{T}
\end{array} .\right.
$$

\section{Roundness of the stem component}

The roundness of the stem is measured using Hough transform [16], which first detects the parameters of a circle when the numbers of all pixels that fall on the objective perimeter are known. Roundness measurements usually use the least square circle, minimum zone circle, maximum inscribed circle, and minimum circumscribed circle. However, the roundness measurement in this study is quantified from the area and perimeter of a two-dimensional image and its value is between 0 and 1, as in Equation (8). Values closer to 1 indicate a greater degree of roundness.

$$
M r=\frac{4 \pi A}{p^{2}}
$$

where $A$ and $p$ are the area and perimeter of the stem, respectively.

\section{Results and discussions}

\section{Spectral Illumination}

The spectrum illumination of white light LED arrays was measured using a spectrometer (SD-1200, OTO Photonics). The electrical performance integration time was $1 \mathrm{~ms}$ to 24 seconds and the slit width of the optics 
was $25 \mu \mathrm{m}$. The spectral resolution and wavelength range of the spectrometer were 1.3 to $5.0 \mathrm{~nm}$ FWHM (full width at half maximum) and 300 to $1000 \mathrm{~nm}$, respectively. White light LEDs in $24 \times 6$ arrays were used in four LED frames. Figure 1 shows the characteristic spectral illumination of the white light LED arrays measured at a horizontal angle. In general, white light is actually a mix of different wavelengths in the visible spectrum from about 380 to $770 \mathrm{~nm}$. Blue light has a clearly higher intensity than other light colors, while red has the lowest intensity. As shown in the CIE chromaticity diagram, blue, red, and green light respectively accounted for $38.04 \%, 30.91 \%$ and $31.05 \%$ of the light source.

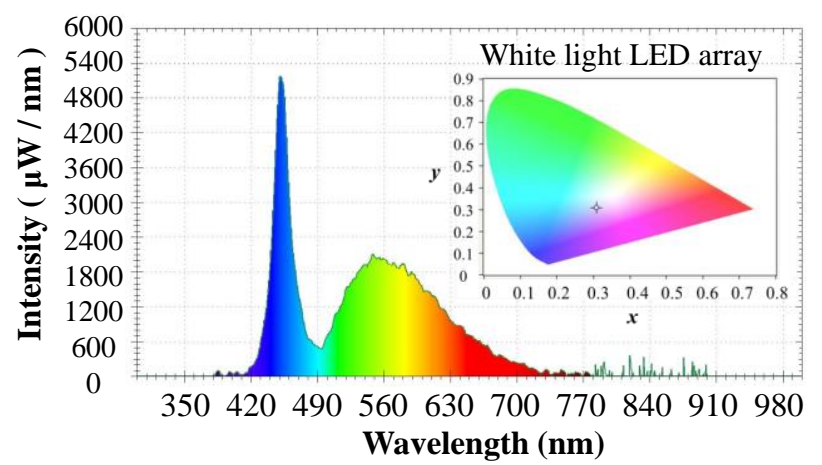

Figure 1. Spectral intensity vs. wavelength of white light LED arrays measured at a horizontal angle.

For the spectral illumination of the light source, a projection angle of light source is defined between a horizontal line and the top LED frame. The measurement range of the illumination characteristics of the light source projection is from 0 to 50 degrees at light illuminations of 645,2240 , and 3840 Lux supplied by the white light LED array. Figure 2 shows that maximum illumination is achieved at an angle of about 20-30 degrees from the object. Significant differences emerge at higher degrees of illumination in white LED arrays.

The illumination characteristics of LED light are important to ensure uniform illumination in the camera's field of view (FOV). The effective illumination range of the white light LED arrays was investigated at different shift displacements. A shift displacement of $0 \mathrm{~cm}$ indicates that the spectrometer is directly under the GigE camera. The measurement range of the shift displacement is from 0 to $3 \mathrm{~cm}$ due to the camera's FOV.

Figure 3 shows the illumination characteristics of the LED lights at different shift displacements for constant light angles of $0,10,30$, and 50 degrees, with corresponding maximum errors of $4.4,4.2,6.8$, and $3.5 \%$. The distribution of the light illumination after shift displacements is more consistent and these errors are below $5 \%$ when the light angle of the LED is $0^{\circ}, 10^{\circ}$ or $50^{\circ}$.



Figure 2. Spectral illumination characteristics of white light LED arrays with different light angles.

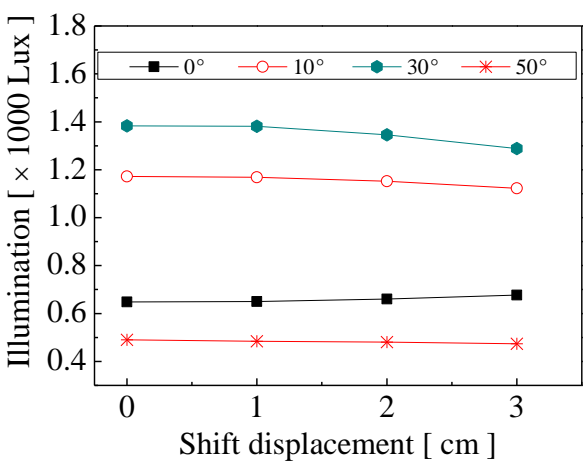

Figure 3. Characteristic illumination of white light LED arrays with differential shift displacements at the $0,10,20,30,40$ and 50 degrees.

\section{Adjusted white-balance}

Figure 4 shows the original image and the white-balance adjusted image of the bicycle stem. The original color image measures $400 \times 350 \times 3$ pixels. The original image has unrealistic color casts and obviously non-neutral colors. The white balance of the cameras is created using the gray world assumption algorithm. The mean values of $R, G$, and $B$ components are 72.8275, 108.0542 and 91.5179 , respectively, with gain factors of $1.2468,0.8403$ and 0.9922 (i.e., dividing the appropriate average for each color component by the gray value). Removing the unrealistic color casts shows a neutral image in the same light after white balance adjustment. The white balance is typically achieved placing GWA algorithm correction filters over the LED lights.

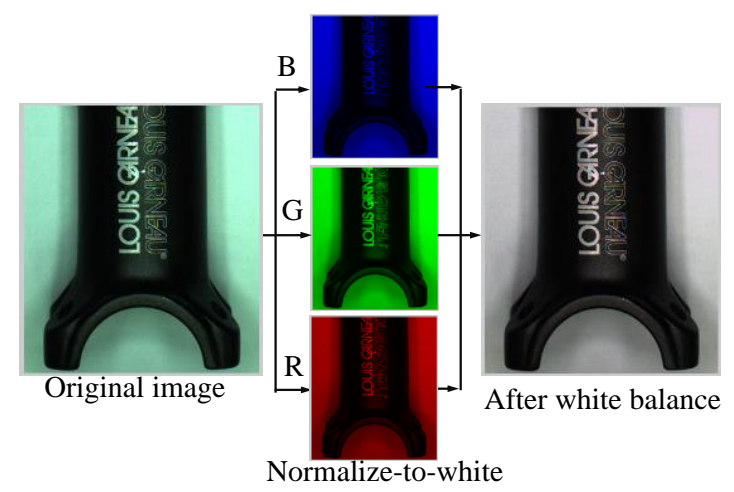

Figure 4. (Left) Original bike stem photo from the digital camera. (Right) Neutral Image using white balance to remove unrealistic color casts. 

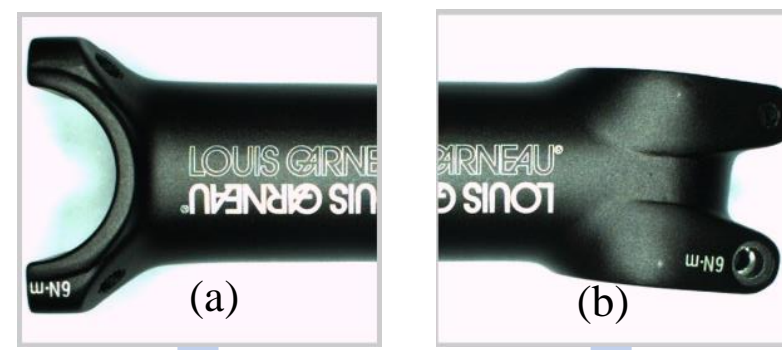

(b)

Keypoint Localization
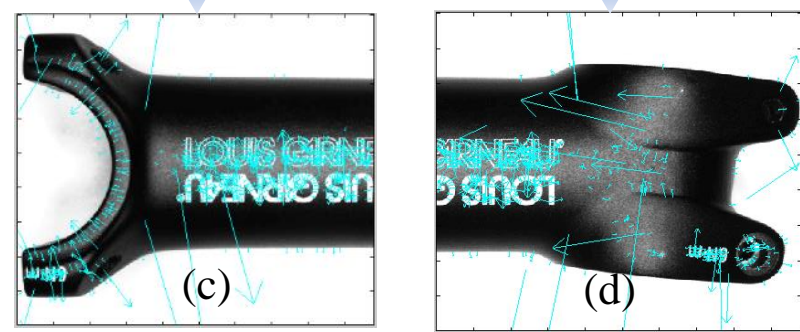



(e)

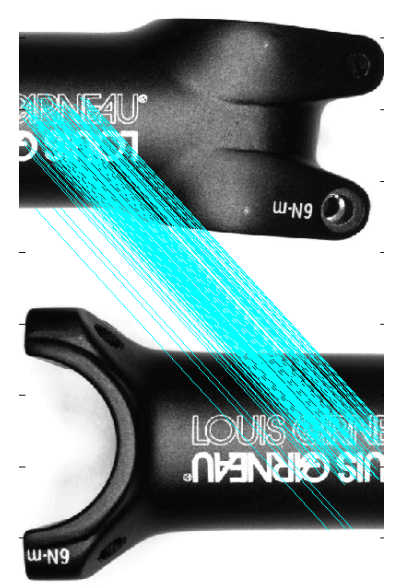

(f)
Figure 5. Image stitching of a bicycle stem: (a) Original image of the top half, (b) original image of bottom half,, (c) and (d) create the gradient directions of each part, (e) SIFT matching and (f) after RANSAC matching.

\section{Scale-invariant feature transforms}

Figures 5(a) and 5(b) respectively show individually captured images for the left-half and right-half sections of the stem. The two images are stitched together to produce a seamless image of the full component using the SIFT algorithm. The geometric dimensions of the complete component are then measured. A histogram of gradient orientations based on the keypoint localization of each part is built from the neighborhood of each interest point, as shown in Figures 5(c) and 5(d). In general, the keypoint localizations have multiple orientations and can significantly improve the reliability of feature matching. The gradient directions at the extremum are useful for rejecting unstable extrema. The SIFT features are insensitive to changes in illumination for the extracted image features, but often fail to match when the features are detected by surface smoothing that casts different shadows under different illuminations. Some failed matches are clearly evident after SIFT stitching, as shown in Figure 5(e). Therefore, a random sample consensus (RANSAC) model is used to provide final verification of whether a set of feature matches corresponds to object. Figure $5(f)$ demonstrates the effectiveness of the hypothesis generation of the RANSAC matching algorithm. RANSAC matching can identify the true correspondence remarkably well, with nearly $99 \%$ matching in the second image. The RANSAC matching results are identified from the gradient directions of each stem part.

After stitching matching, the two images of a stem are joined using SIFT, as shown in Figure 6(a). Using SIFT and RANSAC enables the transformation of the stem image into local feature coordinates that are invariant to illumination changes. In addition, image stitching generates high-quality results for image match verification. For the stem, $L$ is defined as the maximum length of the stem. If the stem image is tilted, the image will be rotated using geometric transformation. Using a micrometer, the original length of the stem was measured as $119 \mathrm{~mm}$. After stitching, the length of the stem is $119.02 \mathrm{~mm}$, for an error of $0.017 \%$, or equivalent to 2 pixels.

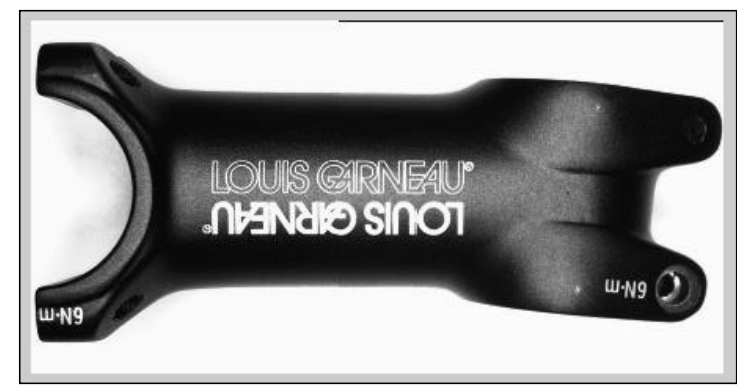

(a)

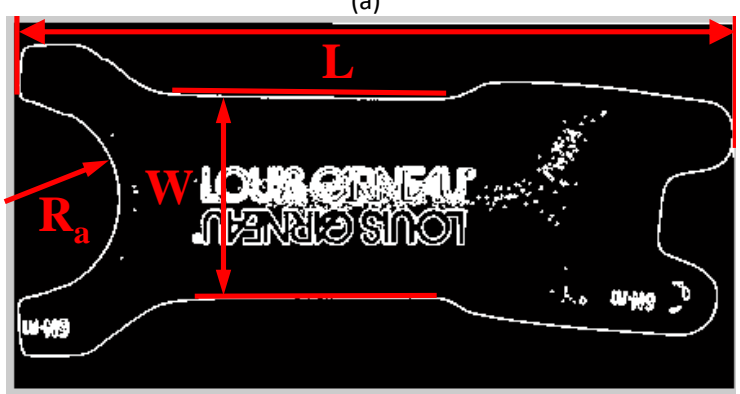

(b)

Figure 6. Bicycle stem (a) after image stitching (b) after image binarization and geometric measurement.

Figure $6(\mathrm{~b})$ shows the binary image of the stem after image stitching. The key geometric measurement dimensions for automatic inspection are the length, width and radius of the stem (119.02, 35.34 and 16.3 $\mathrm{mm}$, respectively). The percentage error for all geometric measurement compared with the original design is $0.017 \%$. Figure 7 shows the roundness measurement process with an open gap for automatic inspection. As 
shown in Figure $7(a)$, the manufacturing process cuts an open gap, which can easily impact the stem's roundness. The roundness measurement is used in non-contact inspection methods to determine deviation in the stem's roundness. As shown in Figures $7(\mathrm{a})$-(d), the roundness inspection is a sequence of processes including: Hough transform, image binarization, definition of a center point, noise filtering based on annular area, edge retrieval using OR logical, image filling and roundness measurement. Results show the center point of the stem is at $(162,152)$ and the roundness is 0.89158 , for an error of $2 \%$ as compared to the roundness error found with contact inspection using a roundness measurement device (ERG-11, Kosaka).

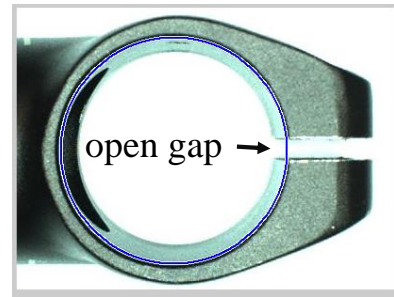

(a)

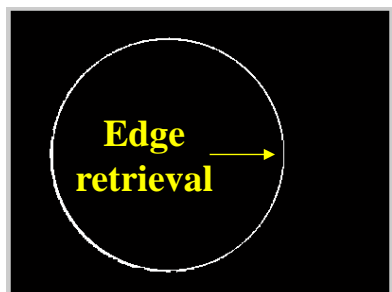

(c)



(b)

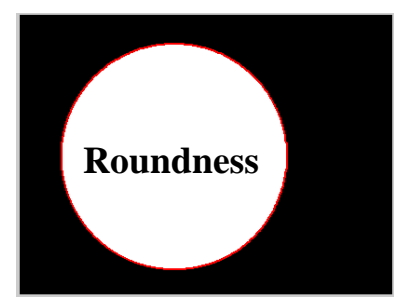

(d)
Figure 7. Roundness measurement of stem accessory (a) round feature detection using Hough transform, (b) noise filtering based on annular area, (c) edge retrieval using OR logical, and (d) roundness measurement.

\section{Conclusion}

This study combines common digital imaging technologies and spectral illumination analysis to quantize light sources during the automatic inspection of a manufactured bicycle component. The illumination characteristics of LED light allow for uniform light distribution in the camera's field of view for the feature inspection of the component. SIFT and RANSAC can transform the image of the object into local feature coordinates that are invariant to changes in illumination. In addition, the image stitching process generates high-quality results for image match verification. White balance, scale-invariant feature transforms, and roundness algorithms are used to implement non-contact automatic inspection of component. This approach can be widely applied in modern industrial manufacturing and provides useful information for automatic inspection and image stitching.

\section{Acknowledgment}

This work was partially supported by the National Science Council of Taiwan, under Grant No. NSC 101-2221- E-150 - 015, and by the J.D. Corporation.

\section{References}

[1] J. Lellmann, B. Lellmann, F. Widmann, and C. Schnörr, "Discrete and continuous models for partitioning problems," International Journal of Computer Vision, vol. 104, no. 3, pp. 241-269, 2013.

doi: $10.1007 / \mathrm{s} 11263-013-0621-4$

[2] S. Samsudin, S. Adwan, H. Arof, N. Mokhtar, and F. Ibrahim, "Development of automated image stitching system for radiographic images," Journal of digital imaging, vol. 26, no. 2, pp. 361-370, 2013.

doi: $10.1007 /$ s10278-012-9483-5

[3] J. Mutch and D. Lowe, "Object class recognition and localization using sparse features with limited receptive fields," International Journal of Computer Vision, vol. 80, no. 1, pp. 45-57, 2008. doi: $10.1007 / \mathrm{s} 11263-007-0118-0$

[4] C. M. Javier, I. Bogdanova, B. Paquier, M. Bierlaire, and J. P. Thiran, "Scale invariant feature transform on the sphere: Theory and applications," International Journal of Computer Vision, vol. 98, no. 2, pp. 217-241, 2012.

doi: $10.1007 / \mathrm{s} 11263-011-0505-4$

[5] J. Jia and C. K. Tang "Image stitching using structure deformation," IEEE Transactions on Pattern Analysis and Machine Intelligence, vol. 30, no. 4, pp. 617-631, 2008.

doi: 10.1109/TPAMI.2007.70729

[6] M. Brown and D. G. Lowe, "Automatic panoramic image stitching using invariant features," International Journal of Computer Vision, vol. 74, no. 1, pp. 59-73, 2007.

doi: $10.1007 / \mathrm{s} 11263-006-0002-3$

[7] W. Liu, Y. Wang, J. Chen, J. Guo, and Y. Lu, "A completely affine invariant image-matching method based on perspective projection," Machine Vision and Applications, vol. 23, no. 2, pp. 231-242, 2012.

doi: $10.1007 / \mathrm{s} 00138-011-0347-7$

[8] T. S. Cho, C. L. Zitnick, N. Joshi, S. B. Kang, R. Szeliski, and W. T. Freeman, "Image restoration by matching gradient distributions," IEEE Transactions on Pattern Analysis and Machine Intelligence, vol. 34, no. 4, pp. 683-694, 2012. doi: $\underline{10.1109 / T P A M I .2011 .166}$ 
[9] Y. Yu, K. Huang, W. Chen, and T. Tan, "A novel algorithm for view and illumination invariant image matching," IEEE Transactions on Image Processing, vol. 21, no. 1, pp. 229-240, 2012. doi: 10.1109/TIP.2011.2160271

[10] F. Orieux, E. Sepulveda, V. Loriette, B. Dubertret, and J. C. Olivo-Marin, "Bayesian estimation for optimized structured illumination microscopy," IEEE transactions on image processing, vol. 21, no. 2, pp. 601-614, 2012. doi: $10.1109 /$ tip. 2011.2162741

[11] M. M. Fouad, R. M. Dansereau, and A. D. Whitehead, "Image registration under illumination variations using region-based confidence weighted m-estimators," IEEE transactions on image processing, vol. 21, no. 3, pp. 1046-1060, 2012.

doi: $10.1109 /$ tip.2011.2167344

[12] C. K. Chow and S. Y. Yuen, "A solution to illumination direction estimation of a shaded image: Genetic algorithm," Image and Vision Computing, vol. 28, no. 12, pp. 1717-1730, 2010. doi: $10.1016 /$ j.imavis.2010.06.001
[13] B. Kim, H. Yoo, and K. Sohn, "Exact order based feature descriptor for illumination robust image matching," Pattern Recognition, vol. 46, no. 12, pp. 3268-3278, 2013.

doi: 10.1016/i.patcog.2013.04.015

[14] D. G. Lowe, "Distinctive image features from scale-invariant keypoints," International Journal of Computer Vision, vol. 60, no. 2, pp. 91-110, 2004.

doi: $10.1023 /$ b:visi.0000029664.99615.94

[15] R. Su, C. Sun, and T. D. Pham, "Junction detection for linear structures based on hessian, correlation and shape information," Pattern Recognition, vol. 45, no. 10, pp. 3695-3706, 2012.

doi: 10.1016/i.patcog.2012.04.013

[16] R. Janssen, M. Verrijt, J. de Best, and R. van de Molengraft, "Ball localization and tracking in a highly dynamic table soccer environment," Mechatronics, vol. 22, no. 4, pp. 503-514, 2012. doi: 10.1016/j.mechatronics.2012.02.009 